\title{
Valoración de características nucleares en muestras citológicas de tumores mamarios caninos
}

\author{
Benítez, J.S.; Insfrán, R.M.; Merlo, W.A.; Brunel, R.A.; Rosciani, A.S. \\ Servicio de Diagnóstico Histopatológico y Citológico, Facultad de Ciencias Veterinarias, Universidad Nacional \\ del Nordeste (UNNE), Cabral 2139, Corrientes (Argentina). E-mail: asrosciani@gmail.com
}

\begin{abstract}
Resumen
Benítez, J.S.; Insfrán, R.M.; Merlo, W.A.; Brunel, R.A.; Rosciani, A.S.: Valoración de características nucleares en muestras citológicas de tumores mamarios caninos. Rev. vet. 27: 2, 117-120, 2016. El presente trabajo tuvo como objetivo comprobar la utilidad y el valor predictivo de la determinación de características morfológicas nucleares subjetivas y objetivas, en muestras citológicas de tumores mamarios caninos. Se estudiaron muestras de 34 pacientes, las cuales fueron controladas hasta 18 meses post cirugía, lo que permitió la confección de curvas de supervivencia. Se realizó la estadificación clínica al momento de la consulta, el citodiagnóstico y la determinación del grado nuclear de las muestras obtenidas por punción y coloreadas con hematoxilina y eosina. Para las mediciones se utilizó el programa Leica Application Suite 3.4.1., calculándose el diámetro nuclear promedio, área nuclear promedio, perímetro nuclear promedio e índice de redondez, todos ellos parámetros cariométricos de valor pronóstico en medicina humana. Los resultados señalaron un aumento gradual de los valores morfométricos de cada parámetro, coincidentes con el aumento de la graduación nuclear, del estadio clínico, así como de los tipos histopatológicos considerados de peor pronóstico. Las curvas de supervivencia mostraron diferencias significativas al comparar los grados nucleares. Los hallazgos efectuados respaldan la cualidad predictiva de estas herramientas diagnósticas.
\end{abstract}

Palabras clave: perra, neoplasias mamarias, citodiagnóstico, graduación nuclear, parámetros cariométricos, valor pronóstico.

\begin{abstract}
Benítez, J.S.; Insfrán, R.M.; Merlo, W.A.; Brunel, R.A.; Rosciani, A.S.: Evaluation of nuclear characteristics in citological samples of canine mammary tumors. Rev. vet. 27: $2,117-120,2016$. This work aimed to validate the usefulness of subjective and objective determination of nuclear morphological characteristics in cytological samples of canine mammary tumors and check their predictive utility. Samples of 34 patients who were followed up for 18 months post surgery, were studied, situation that allowed survival curves confection. Clinical staging at diagnosis was made. The samples were obtained by fine needle punction, colored with hematoxylin and eosin and cytologic diagnosis and nuclear graduation were performed. The Leica Application Suite 3.4.1 Program was used for measurements, calculating the average nuclear diameter, the average nuclear area, the average nuclear perimeter and roundness index. All of these nuclear morphometric parameters are considered of prognostic value in human medicine. In this analysis, a gradual increase in morphometric values of each parameter was observed. In accordance with higher nuclear graduation and clinical stages and also, in histological types considered of worse prognosis. Survival curves showed statistical differences when comparing nuclear degrees. The findings obtained here, support the predictive quality of determination of nuclear grade and its morphometric parameters.
\end{abstract}

Key words: bitch, mammary neoplasias, cytologic diagnosis, nuclear graduation, cariometric parameters, prognosis value.

\section{INTRODUCCIÓN}

Las neoplasias mamarias constituyen los tumores más frecuentes de las hembras caninas y son llamativas

Recibido: 7 abril 2016 / Aceptado: 22 julio 2016 por su gran diversidad histomorfológica y su comportamiento biológico variable, difícil de predecir. Especialistas en el tema afirman que el reconocimiento de neoplasias verdaderamente malignas es problemático $\mathrm{y}$ que se tiende a sobrediagnosticar estas lesiones. Por ello recomiendan que al evaluar cortes histopatológi- 
cos con hematoxilina y eosina, se tenga en cuenta el tipo histológico, el grado de pleomorfismo celular y nuclear, el índice mitótico, las áreas de necrosis dentro del tumor, la invasión vásculo-linfática intra y peritumoral y la presencia de metástasis ganglionar ${ }^{8}$.

Basándose en una clasificación que reveló idoneidad en pacientes humanos ${ }^{7}$, se han propuesto varios sistemas de graduación de malignidad para estas neoplasias ${ }^{4,8,15}$. La disparidad de criterios existentes en los sistemas de graduación morfológica del cáncer mamario en caninos, genera una amplia variedad de conceptos y metodologías al momento de elaborar el diagnóstico y el pronóstico de vida para las pacientes. Ello demuestra la necesidad de investigar nuevos métodos objetivos confiables y reproducibles.

El estudio citológico constituye una herramienta eficaz en la aproximación diagnóstica de numerosos tipos de lesiones, facilitando la apreciación de las características nucleares. El grado de diferenciación nuclear de las células neoplásicas (grado nuclear), se viene utilizando desde hace mucho tiempo para diferenciar entre lesiones benignas y malignas, así como para determinar el riesgo de progresión de la enfermedad ${ }^{16}$. Sin embargo, en general, carece de objetividad y de reproducibilidad debido a las variaciones propias de cada observador ${ }^{9,14}$.

Para compensar estas falencias se ha propuesto la aplicación de métodos morfométricos que exigen estandarización de los procedimientos de obtención y preparación de las muestras a valorar, del manejo de muestras representativas de una determinada lesión o tipo celular y de la selección del área del espécimen a medir ${ }^{18}$.

Entre los numerosos parámetros estudiados, el más común es el área nuclear, determinada por el contorno nuclear a partir del cual pueden calcularse otros parámetros morfométricos como el diámetro, el perímetro y el indice de redondez nuclear ${ }^{1}$. Este último refleja la forma del núcleo celular, consignándose el valor de uno (1), cuando la misma se corresponde con un círculo perfecto ${ }^{19}$.

En medicina veterinaria diversos estudios en diferentes tipos de neoplasias han recurrido al análisis de la morfometría nuclear como predictor ${ }^{5,9}$. Se ha propuesto la utilidad diagnóstica y predictiva que provee la cuantificación de estas características en las células de neoplasias mamarias de la mujer, lo cual podría ayudar a discriminar diferentes tipos de lesiones, evaluar el curso de la enfermedad, la supervivencia, el riesgo de metástasis e incluso discriminar la posibilidad de respuesta al tratamiento quimioterápico ${ }^{1}$.

Aunque el examen histopatológico continúa siendo el método más confiable para el diagnóstico ${ }^{12}$, el potencial, la eficacia y la practicidad clínica que representa la aplicación del citodiagnóstico, han sido comprobados hace tiempo en medicinas humana y veterinaria ${ }^{11}$. En la evaluación del carcinoma mamario de la mujer, la valoración morfométrica de diferentes parámetros nucleares aporta una valiosa información pronóstica adicional ${ }^{18}$.
El presente trabajo tuvo como objetivo comprobar la utilidad predictiva de la determinación de las características morfológicas nucleares medibles en muestras citológicas de tumores mamarios caninos.

\section{MATERIAL Y MÉTODOS}

Se trabajó con 34 pacientes caninas con neoplasias mamarias, cuya evolución pudo controlarse hasta los 18 meses. En el caso de aquéllas que presentaron múltiples tumoraciones, se estudió la progresión del tumor de mayor dimensión.

Se tomaron muestras citológicas de los tumores mamarios por el método de "punción con aguja fina", variante de la "punción-aspiración con aguja fina" ${ }^{2,3}$. Se utilizaron agujas descartables 40 x 8 y jeringas de $10 \mathrm{ml}$, para expeler el material sobre portaobjetos limpios y desengrasados. Los preparados fueron fijados en alcohol de $96^{\circ}$ durante 5 minutos y coloreados con hematoxilina y eosina.

El diagnóstico citológico se realizó en base a una clasificación y graduación histológica de tumores mamarios del perro ${ }^{8}$. Se procedió a la determinación de la graduación nuclear (GN), de acuerdo al ítem correspondiente a "pleomorfismo nuclear" del "sistema de graduación histológica de los carcinomas mamarios caninos y felinos" ${ }^{13}$. En base al mismo, las pacientes fueron agrupadas en tres categorías (GN I, II y III), teniendo en cuenta el pleomorfismo, la condensación de la cromatina y la presencia y tamaño del nucleolo.

Se obtuvieron fotografías digitales utilizando un microscopio Leica DM500 con cámara digital Leica ICC50 acoplada. Sobre las imágenes se determinaron los valores de área nuclear promedio, perímetro nuclear promedio, diámetro nuclear promedio e índice de redondez (perímetro nuclear $2 / 4 \pi$. área nuclear). Se registraron los valores de los recuentos realizados en 50 células de cada caso, utilizando el Leica Application Suite 3.4.1. Todas las mediciones se hicieron bajo un aumento de 400X y se expresaron en micrómetros.

Se procedió a la determinación del estadio clínico de las pacientes (Owen, 1980) considerando la clasificación "TNM" de la OMS, la cual contempla cinco categorías, de menor a mayor gravedad: I, II, III, IV y V. Los controles post-tratamiento fueron realizados hasta el año y medio, luego del tratamiento quirúrgico.

En base a las mencionadas determinaciones se confeccionaron "curvas de sobrevida de Kaplan-Meier". Estas curvas muestran en la ordenada, la probabilidad (que decrece desde 1) que tienen las pacientes con neoplasias mamarias de sobrevivir determinada cantidad de tiempo, representada en meses en la abscisa. Se buscaron diferencias entre los grupos considerados para cada uno de los parámetros estudiados, por medio de pruebas de $\chi^{2}$ y análisis de la variancia. Las diferentes variables fueron analizadas mediante el programa $I n$ foStat Profesional, versión 10 (FCA, Universidad Nacional de Córdoba) ${ }^{6}$. 


\section{RESULTADOS}

Las neoplasias estudiadas fueron diagnosticadas como diferentes variedades malignas de carcinomas (simples: 10 casos; complejos: 13 casos; mixtos: 8 casos) y sarcomas en 3 ocasiones.

La distribución de las neoplasias en las distintas categorías contempladas en la graduación nuclear, se observa en la Tabla 1.

Los valores promedio obtenidos para los distintos parámetros cariométricos, teniendo en cuenta la estadificación clínica, el diagnóstico del tipo histológico y la graduación nuclear, se detallan en la Tabla 2. La comparación de dichos parámetros entre las categorías de la estadificación clínica y del tipo histológico, no arrojó diferencias significativas, aunque sí las hubo (p: $0,0001)$ al comparar las tres categorías de la graduación nuclear.

Las "curvas de sobrevida" revelaron una disminución significativa (p: 0,002) del tiempo de supervivencia en las categorías de mayor graduación nuclear, tal como puede apreciarse en la Figura 1. No se obtuvieron diferencias significativas al analizar la supervivencia de las pacientes considerando la estadificación clínica o el tipo histológico.

Tabla 1. Distribución de las pacientes de acuerdo a la graduación nuclear.

\begin{tabular}{lcc}
\hline grado nuclear & $\mathrm{n}$ & $\%$ \\
\hline I & 11 & 32,3 \\
II & 16 & 47,0 \\
III & 7 & 20,6 \\
\hline
\end{tabular}

n: número de casos; \%: porcentajes sobre el total de casos analizados.

Tabla 2. Parámetros cariométricos según estadificación clínica (EC), tipo histológico (TH) y grado nuclear (GN).

\begin{tabular}{llcccc}
\hline tipo & grado & DM $\mu \mathrm{m}$ & ${\mathrm{AM} \mu \mathrm{m}^{2}}^{2}$ & $\mathrm{PM} \mu \mathrm{m}$ & $\mathrm{IR}$ \\
\hline \multirow{4}{*}{ EC } & I & 9,53 & 84,04 & 29,93 & 0,95 \\
& II & 8,78 & 78,21 & 29,41 & 0,94 \\
& III & 9,96 & 86,82 & 30,92 & 0,95 \\
& IV & 10,89 & 92,92 & 34,47 & 0,97 \\
& V & 11,34 & 103,82 & 31,47 & 0,97 \\
\hline \multirow{4}{*}{ TH } & CC & 9,24 & 83,04 & 29,73 & 0,95 \\
& CTM & 9,42 & 87,72 & 31,28 & 0,95 \\
& CS & 10,62 & 91,72 & 32,24 & 0,95 \\
& SAR & 11,04 & 94,52 & 32,27 & 0,97 \\
\hline \multirow{3}{*}{ GN } & 1 & 7,99 & 60,19 & 25,7 & 0,92 \\
& 2 & 9,94 & 87,33 & 30,44 & 0,95 \\
& 3 & 10,87 & 102,07 & 34,37 & 0,97 \\
\hline
\end{tabular}

DM: diámetro nuclear promedio; AM: área nuclear promedio; PM: perímetro nuclear promedio; IR: índice de redondez; I, II, III, IV y V: estadificación clínica; CS: carcinoma simple; CC: carcinoma complejo; CTM: carcinoma en tumor mixto; SAR: sarcoma; 1, 2 y 3: grado nuclear en sus tres categorías.

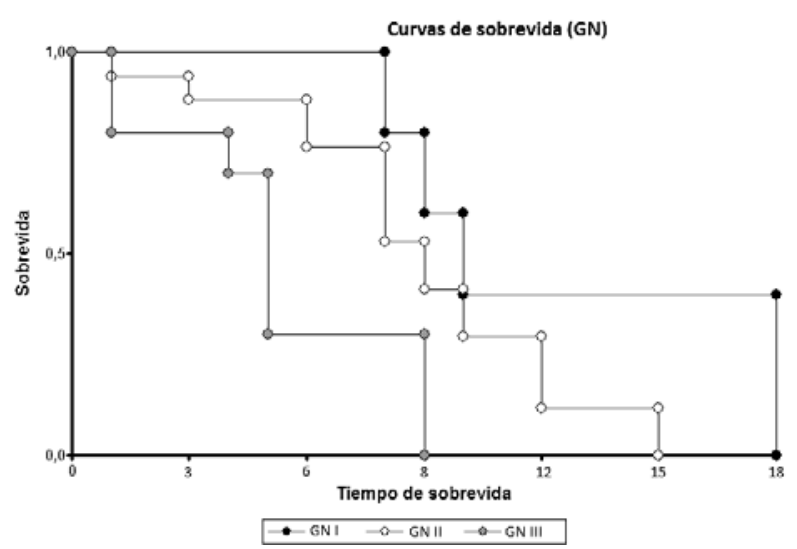

Figura 1. Curva de sobrevida de acuerdo con la graduación nuclear (GN). GN I, GN II y GN III Abscisa: sobrevida (meses). Ordenada: probabilidad de sobrevida.

\section{DISCUSIÓN}

Todas las muestras analizadas fueron diagnosticadas como variedades malignas de neoplasias mamarias. La citología demostró el potencial atribuido en medicina humana como técnica diagnóstica complementaria ${ }^{11}$, siendo aplicable en muestras citológicas de tumores mamarios caninos, acorde a lo demostrado por otros autores ${ }^{5}$.

Los valores morfométricos nucleares de todos los parámetros considerados, mostraron un incremento progresivo en relación directa al aumento de la graduación nuclear y de la estadificación clínica considerada. Aunque no se encontraron diferencias significativas entre los distintos tipos histológicos, los valores más elevados se observaron en el grupo de los sarcomas, considerados por la bibliografía clásica como los de peor pronóstico. Estos resultados son coincidentes con los hallazgos efectuados en carcinomas mamarios de la mujer ${ }^{17}$, que indican en forma cuantitativa los cambios de forma y de tamaño que sufre el núcleo celular durante el proceso de malignización.

Si bien el índice de redondez mostró pequeñas fluctuaciones, en ningún caso alcanzó el valor de 1, lo que indicaría que el núcleo de las células tumorales del canino conserva una forma ovoide, tal como sucede en el carcinoma mamario de la mujer ${ }^{10}$. La menor supervivencia de las pacientes con índices próximos a dicho valor coincide con la premisa de que la aproximación a la esfericidad conlleva un mal pronóstico ${ }^{19}$.

Los resultados de la valoración de las características morfológicas y morfométricas nucleares en muestras citológicas y su correlación con la sobrevida de las pacientes afectadas por neoplasias mamarias, coinciden con la idea expresada por investigadores que consideran a estas determinaciones como instrumentos complementarios eficaces para predecir la evolución de las pacientes ${ }^{1}$. El uso de programas informáticos analizadores de imágenes constituye una herramienta 
eficaz en el área, ya que facilita la apreciación y medición de ciertas características citológicas, permitiendo obtener resultados cuantificables y reproducibles que podrían resultar de utilidad para definir parámetros estandarizados, aún inexistentes en medicina veterinaria.

El citodiagnóstico de neoplasias mamarias caninas, teniendo en cuenta la graduación nuclear subjetiva y la determinación de parámetros morfométricos nucleares objetivos, se muestran en estos resultados como herramientas diagnósticas con valor pronóstico acerca de la sobrevida de las pacientes.

\section{REFERENCIAS}

1. Abdalla F, Boder J, Markus R, Hashmi H, Buhmeida A, Collan Y. 2009. Correlation of nuclear morphometry of breast cancer in histological section with clinicopathological features and prognosis. Anticancer Res 29: 1771-1776.

2. Akhtar M, Ashraf A, Huq M, Faulkner C. 1989. Fineneedle biopsy: comparison of cellular yield with and without aspiration. Diagn Cytopathol 5: 162-165.

3. Cajulis R, Sneige N. 1993. Objective comparison of cellular yield in fine-needle biopsy of lymph nodes with and without aspiration. Diagn Cytopathol 9: 43-45.

4. Clemente M, Perez MD, Illera JC, Peña L. 2010. Histologic, immunologic and ultrastructural description of vasculogenic mimicry in canine mammary cancer. Vet Pathol 47: 265-274.

5. De Vico G, Sfacteria A, Maiolino P, Mazzullo G. 2002. Comparison of nuclear morphometric parameters in cytologic smears and histologic sections of spontaneous canine tumors. Vet Clin Pathol 31: 16-18.

6. Di Rienzo JA, Casanoves F, Balzarini MG, Gonzalez L, Tablada M, Robledo CW. 2011. InfoStat versión 2011. GrupoInfoStat, FCA, Universidad Nacional de Córdoba, Argentina. http://www.infostat.com.ar

7. Elston CW, Ellis IO. 1991. Pathological prognostic factors in breast cancer, I: The value of histological grade in breast cancer: experience from a large study with longterm follow-up. Histopathol 19: 403-410.

8. Goldschmidt M, Peña L, Rasotto R, Zappulli V. 2011. Classification and grading of canine mammary tumors. Vet Pathol 48: 85-97.

9. Martano M, Damiano S, Restucci B, Paciello O, Russo V, Maiolino P. 2006. Nuclear morphometry in canine acanthomatous ameloblastomas and squamous cell carcinomas. Eur J Histochem 50: 125-130.
10. Martinez R, García MT, Sampedro A. 2000. Grado nuclear en punciones de carcinoma de mama: características ópticas y análisis de imagen. Rev Esp Patol 32: 139-145.

11. Masood S. 2000. Assessment of prognostic factors in breast fine-needle aspirates. Am J Clin Pathol 113: S84S96.

12. Misdorp W, Else RW, Hellmen E, Lipscomb TP. 1999. Histological classification of mammary tumors of the dog and the cat, Publ. by Armed Forces Institute of Pathology, 2nd series, Vol. VII, Washington DC (USA), 58 p.

13. Misdorp W. 2002. Tumors of the mammary gland. In: Tumors in domestic animals (Meuten DJ, ed.), $4^{\circ}$ ed., Iowa State Press, Ames (USA), p. 575-606.

14. Parada D, Farias R, García J. 1999. Análisis morfométrico de variables en núcleos de células normales y neoplásicas de la glándula mamaria: informe preliminar. Invest Clin (Venezuela) 40: 233-244.

15. Peña L, De Andrés PJ, Clemente $M$, Cuesta P, PérezAlenza MD. 2013. Prognostic value of histological grading in non inflammatory canine mamary carcinomas in a prospective study with two-year follow-up: Relationship with clinical and histological characteristics. Vet Pathol 50: 94-105.

16. Simeonov R, Simeonova G. 2006. Use of nuclear roundness as a complementary method for differentiation of benign from malignant canine mammary gland epithelial tumour on cytologic smears. Trakia J Sci (Bulgaria) 4: 4750 .

17. Torres FJ, Calle LF, Torres FJ. 2008. Correlación entre parámetros cariométricos, estatus ganglionar y expresión inmunohistoquímica en el carcinoma ductal infiltrante mamario. Rev Esp Patol 41: 189-194.

18. Torres FJ, Calle LF, Torres FJ. 2009. Correlación entre la valoración subjetiva de parámetros nucleares en la PAAF, del grado citológico y del grado histológico y la morfometría, en el carcinoma ductal infiltrante mamario. Rev Esp Patol 42: 25-30.

19. Van der Linden HC, Baak JP, Lindeman J, Hermans J, Meyer CJ. 1986. Morphometry and breast cancer. II: Characterization of breast cancer cells with high malignant potential in patients with spread to lymph nodes: Preliminary results. J Clin Pathol 39: 603-609. 